\title{
A Bayesian Assessment of the Longevity of Jeanne Calment
}

\author{
Nikolay Zak ${ }^{1}$ and Philip Gibbs ${ }^{2}$
}

\begin{abstract}
Mme Calment died in 1997 at the reputed age of 122 years, the longest validated life span of all time. Recently it has been suggested that in fact Mme Calment was not Jeanne Calment born in 1875 as believed, but rather her daughter Yvonne born in 1898. She could have swapped identity on her mother's death in 1934. In this study, the most reliable evidence is evaluated and competing versions of hypothetical scenarios are compared. No information is completely certain so only probabilities are considered. Methods of Bayesian inference are used to assess the probability of correctness for each scenario. It is not possible to form a definitive conclusion with the evidence currently available, but we believe that the outcome reinforces earlier suggestions that Mme Calment's age validation is unsafe in the light of recent evidence and should be reviewed. Indicators from DNA analysis that could reliably resolve the question of whether or not an identity switch took place are provided. The methods of Bayesian inference used here could be applied to other longevity validations to improve the validation process for longevity.
\end{abstract}

Keywords: longevity, supercentenarian, France, Bayesian, tuberculosis, DNA, Jeanne Calment, validation

\section{Introduction}

$\mathbf{T}$ He SCIENTIFIC Validation of the life span of centenarians and supercentenarians has become an important cornerstone of gerontological research. Errors in longevity records at extreme ages are not exceptional, so reliable demographic data depend on carefully checking the evidence to eliminate or correct any false records. ${ }^{1,2}$ The official world record for validated longevity is held by Jeanne Calment who died in 1997 with the reputed age of 122 years and 164 days. ${ }^{3}$ Recently this record has been challenged by investigators who claim that her daughter Yvonne Calment made a switch to take over her mother's identity on her death from tuberculosis (TB) in $1934 .^{4-6}$

Jeanne Calment's longevity is significantly longer than any other validated case. The question of her authenticity is an important scientific matter with a bearing on the nature of aging. In addition to her age being a significant data point in its own right, the method of her validation is considered a gold standard. If it is shown to have failed, then other validated cases of extreme age may need to be revisited.

Publicity over the claims against Jeanne Calment has led to a counter investigation resulting in the discovery of further evidence and the release of previously unseen photographs and letters by some of Mme Calment's relatives. This has enabled us to gain a clearer idea of her life and what might have taken place.
Our goal here is to evaluate the ID switch hypothesis using probabilistic methods. A naive methodology based on the improbability of Jeanne Calment's age within her cohort would risk falling foul of the prosecutor's paradox or base rate bias. To avoid these pitfalls, we use the methods of Bayesian inference. Hypothetical scenarios that best represent the most probable possibilities for what might have happened under the switch and no switch options are proposed. They are then compared probabilistically with the most reliable pieces of evidence. The conclusion is subject to uncertainty, but we believe that it gives a better representation of how evidence should be evaluated in ambiguous cases. Further emerging evidence could still change the analysis. In particular, DNA testing is likely to be near conclusive if DNA can be made available and legal hurdles can be overcome. It is understood that suitable blood samples taken from Jeanne Calment are kept by the Jean-Dausset foundation in Paris, and that Mme Calment was always happy to submit to scientific tests.

This report begins with a formal discussion of how the methods of Bayesian inference can be applied to longevity validation in a case such as that of Jeanne Calment. This is followed by a summary of new evidence, some of which has been collected by members of the Facebook group "Contre Enquete Sur L'Enquete Jeanne Calment." The discussion group was created by people from Arles, Jeanne Calment's hometown. They are interested in confirming the validation of Jeanne Calment's age. ${ }^{7}$ We thank them for their investigative

\footnotetext{
${ }^{1}$ Department of Gerontology, Moscow Society of Naturalists, Moscow, Russia.

${ }^{2}$ Independent Scholar, Langdon Hills, Essex, United Kingdom.
} 
work and for allowing those of us who are more skeptical to work with them. They are due the credit for some of the evidence used in this article and many of the discoveries made from them, but the interpretation and analysis are ours.

From this point forward, the elderly lady who died in Arles in 1997 is referred to as simply Mme Calment. Where Jeanne Calment is named, it refers to the person known to have been born in 1875 with that name. The goal is to address the question of whether Mme Calment was Jeanne Calment or her daughter Yvonne Calment who was born in 1898 and whose death from pleurisy was recorded in 1934. The possibility that there was an identity switch between mother and daughter before or at that moment remains a hypothesis. No accusations of wrongdoing are being made. If the switch did take place, it is likely that very few people knew about it. Even Mme Calment's close family could have been unaware. The full circumstances, motivations, and any mitigation would remain unknown. It is not the goal of this report to judge anyone or to determine who may or may not have been complicit if the ID switch took place. Our detailed switch scenarios are hypothetical.

The probability of the hypothesis of the ID switch is evaluated against the available evidence. Here "evidence" means any information that, when taken into account, can affect the probability for or against the identity switch hypothesis. We urge caution when reading automated translations of this document into French. The words "evidence" and "proof" may both be translated as "preuve" despite having very different meanings in English. Nothing we know at present can be regarded as clear proof either way. We expect that further evidence will continue to emerge, which could improve our understanding of possible scenarios and may change the balance of probability. Any conclusions here are therefore preliminary.

Many pieces of useful evidence previously proposed in favor of the ID switch by Nikolay Zak and others are not explicitly taken into account here. ${ }^{4,5}$ This includes much of the evidence based on the testimony of Mme Calment. Some testimonial evidence has also been used to support the validation of Mme Calment's age. If this evidence were to be taken into account, it could affect the likelihood ratio in the Bayesian test by a small factor either way, but the overall conclusion would not change.

It is our opinion that matching the identity of people in different photographs is too uncertain without distinguishing features or careful biometric measurements. As more photographs have been found, it has become clear that over time Jeanne Calment and her daughter Yvonne Calment looked more alike than previously thought. Also, estimations of age based on appearance can easily be subject to surprisingly large errors, especially when taken from low-resolution black and white photographs that are shot under uncontrolled lighting conditions. A person's estimated age is often based unduly on the amount of gray or white hair one has. We have particular reason to mistrust this indicator in the case of Mme Calment. For these reasons, we stick to only the most reliable items of evidence for the formal analysis.

\section{Methodology of Bayesian Inference}

Consider a person $C$ who lived until a recorded age at death of $J_{D}$ according to a base scenario $\boldsymbol{J}$. An alternative hypothesis given by a scenario $\boldsymbol{Y}$ suggests that if some unusual events took place such as an identity switch or misinformation about the age from some point, then $C$ may have lived only to a younger age $Y_{D}<J_{D}$. The longevity of $C$ needs to be validated against the known evidence $\boldsymbol{E}$.

In the case of Mme Calment, $\boldsymbol{J}$ is the scenario that she was indeed Jeanne Calment who lived to an age $J_{D}=122$. $Y$ is the scenario consistent with the switch hypothesis according to which Mme Calment was really Yvonne who died at age $Y_{D}=99$.

The set of all possible histories forms a probability space $\Omega$ with a probability measure $p$ (Figure 1). A scenario $\boldsymbol{H}$ is determined by a subset $\boldsymbol{H} \subset \boldsymbol{\Omega}$ assigning a prior probability $p(\boldsymbol{H})$. The evidence determines a subspace $\boldsymbol{E} \subset \Omega$ of all histories, which are consistent with everything known about the case.

The probability for a hypothesis $\boldsymbol{H}$ given the evidence $\boldsymbol{E}$ is the conditional probability.

$$
p(\boldsymbol{H} \mid \boldsymbol{E})=\frac{p(\boldsymbol{H} \cap \boldsymbol{E})}{p(\boldsymbol{E})}
$$

Validation of extreme ages requires a chaining of events that is devoid of ambiguity. ${ }^{3}$ A potential ambiguity exists if an opportunity for an identity switch or age change can be identified. It is not useful to consider the probability of someone reaching an age of 122 years in isolation. Knowing the probability $p(\boldsymbol{J} \mid \boldsymbol{E})$ of the base scenario does not help validate the longevity of $C$ on its own, even if we could compare it with the cohort sample size. Instead we must calculate a confidence level using the likelihood ratio $r(\boldsymbol{Y}, \boldsymbol{J}, \boldsymbol{E})$ against any viable alternative hypothesis.

$$
r(\boldsymbol{Y}, \boldsymbol{J}, \boldsymbol{E})=\frac{p(\boldsymbol{Y} \mid \boldsymbol{E})}{p(\boldsymbol{J} \mid \boldsymbol{E})}=\frac{p(\boldsymbol{Y} \cap \boldsymbol{E})}{p(\boldsymbol{J} \cap \boldsymbol{E})}
$$

If $r(\boldsymbol{Y}, \boldsymbol{J}, \boldsymbol{E})<c(C) \ll 1$ for any alternative hypothesis, then there is no ambiguity in the evidence and $C$ is considered validated with confidence level $c(C)$.

There will always be relevant facts about the life of $C$ that we will never know. It may therefore be useful or necessary to consider a number of mutually exclusive subscenarios $\boldsymbol{Y}^{(i)}$ for what might have happened, for example, for the alternative scenario.

$$
\begin{gathered}
\boldsymbol{Y}=\bigcup_{i} \boldsymbol{Y}^{(i)} \\
p(\boldsymbol{Y} \cap \boldsymbol{E})=\sum_{i} p\left(\boldsymbol{Y}^{(i)} \cap \boldsymbol{E}\right)
\end{gathered}
$$

It is not possible to check all possible subscenarios for the switch hypothesis, but for any one subscenario $\boldsymbol{Y}^{(i)}$ an inequality holds,

$$
r(\boldsymbol{Y}, \boldsymbol{J}, \boldsymbol{E}) \geq \frac{p\left(\boldsymbol{Y}^{(i)} \cap \boldsymbol{E}\right)}{p(\boldsymbol{J} \cap \boldsymbol{E})}
$$

This means that if we can find one subscenario with a high enough probability, then we can set a lower limit on the likelihood ratio. It does not matter that we have insufficient evidence to say that this subscenario is the right one. If any possible subscenario gives a lower limit $>1$, we can 
conclude that it is not possible to validate $C$ without additional evidence. This is what we propose to do in the case of Mme Calment.

The scenarios (or subscenarios) $\boldsymbol{H}$ (which can be $\boldsymbol{Y}^{(i)}$ or $\boldsymbol{J}$ ) and evidence $\boldsymbol{E}$ can be broken down into individual events or facts $\boldsymbol{H}_{i}$, which are the components of the evidence or subscenarios. For convenience, they are combined into one ordered sequence for each scenario. Facts from the evidence are common to any scenario sequence, while facts from the scenarios or subscenarios can vary. In our formalism, an individual fact $\boldsymbol{H}_{i}$ is identified with the subspace of $\Omega$ that is consistent with that fact.

$$
\boldsymbol{H} \cap \boldsymbol{E}=\bigcap_{i} \boldsymbol{H}_{i}
$$

When the facts are independent, then the probabilities for each can be multiplied, but this is often not the case. More generally, conditional probabilities must be used. The overall probability is the product of the probability of individual facts conditional on its preceding facts.

$$
p\left(\bigcap_{i} \boldsymbol{H}_{i}\right)=\prod_{i} p\left(\boldsymbol{H}_{i} \mid \bigcap_{j<i} \boldsymbol{H}_{j}\right)
$$

Any ordering of the facts can be used in this product breakdown. Chronological order is often more intuitive, but there can be exceptions where posterior evidence provides a probable constraint on the events of the past. From this we form our main lemma, which is used for Bayesian inference.

Lemma. Let $\boldsymbol{Y} \subset \Omega$ be the histories in the base scenario for validation, $\boldsymbol{J} \subset \Omega$ the histories in an alternative scenario, and $\boldsymbol{E} \subset \Omega$ the histories consistent with the evidence. The scenarios are combined with the evidence and broken down into an ordered sequence of $n$ corresponding facts

$$
\boldsymbol{Y} \cap \boldsymbol{E}=\bigcap_{i \leq n} \boldsymbol{Y}_{i}, \quad \boldsymbol{J} \cap \boldsymbol{E}=\bigcap_{i \leq n} \boldsymbol{J}_{i}
$$

Then the likelihood ratio for the alternative scenario verses the base scenario is given by the following:

$$
r(\boldsymbol{Y}, \boldsymbol{J}, \boldsymbol{E})=\frac{p(\boldsymbol{Y} \cap \boldsymbol{E})}{p(\boldsymbol{J} \cap \boldsymbol{E})}=\prod_{i \leq n} R_{i}
$$

where

$$
R_{i}=\frac{p\left(\boldsymbol{Y}_{i} \mid \bigcap_{j<i} \boldsymbol{Y}_{j}\right)}{p\left(\boldsymbol{J}_{i} \mid \bigcap_{j<i} \boldsymbol{J}_{j}\right)}=\frac{p_{i}(\boldsymbol{Y})}{p_{i}(\boldsymbol{J})}
$$

The quantities $p_{i}(\boldsymbol{H})=p\left(\boldsymbol{H}_{i} \mid \bigcap_{j<i} \boldsymbol{H}_{j}\right)$ are conditional weights for facts $\boldsymbol{H}_{i}$ in a scenario $\boldsymbol{H}$. These quantities need to be estimated or calculated from statistical data.

Proof. Define a partial likelihood ratio

$$
r_{k}(\boldsymbol{Y}, \boldsymbol{J}, \boldsymbol{E})=\frac{p\left(\bigcap_{i \leq k} \boldsymbol{Y}_{i}\right)}{p\left(\bigcap_{i \leq k} \boldsymbol{J}_{i}\right)}
$$

It will be shown by induction on $k$ that

$$
r_{k}(\boldsymbol{Y}, \boldsymbol{J}, \boldsymbol{E})=\prod_{i \leq k} R_{i}, \quad 1 \leq k \leq n
$$

$$
\text { For } \begin{aligned}
k=1, r_{1}(\boldsymbol{Y}, \boldsymbol{J}, \boldsymbol{E}) & =\frac{p\left(\bigcap_{i \leq 1} \boldsymbol{Y}_{i}\right)}{p\left(\bigcap_{i \leq 1} \boldsymbol{J}_{i}\right)}=\frac{p\left(\boldsymbol{Y}_{1}\right)}{p\left(\boldsymbol{J}_{1}\right)}=\frac{p\left(\boldsymbol{Y}_{1} \mid \Omega\right)}{p\left(\boldsymbol{J}_{1} \mid \Omega\right)} \\
& =\frac{p\left(\boldsymbol{Y}_{1} \mid \bigcap_{i<1} \boldsymbol{Y}_{i}\right)}{p\left(\boldsymbol{J}_{1} \mid \bigcap_{i<1} \boldsymbol{J}_{i}\right)}=R_{1}=\prod_{i \leq 1} R_{i}
\end{aligned}
$$
then

Now assume for $k \geq 2$ that $r_{k-1}(\boldsymbol{Y}, \boldsymbol{J}, \boldsymbol{E})=\prod_{i<k-1} R_{i}$

$$
r_{k}(\boldsymbol{Y}, \boldsymbol{J}, \boldsymbol{E})=\frac{p\left(\bigcap_{i \leq k} \boldsymbol{Y}_{i}\right)}{p\left(\bigcap_{i \leq k} \boldsymbol{J}_{i}\right)}=\frac{p\left(\boldsymbol{Y}_{k} \cap\left(\bigcap_{i \leq k-1} \boldsymbol{Y}_{i}\right)\right)}{p\left(\boldsymbol{J}_{k} \cap\left(\bigcap_{i \leq k-1} \boldsymbol{J}_{i}\right)\right)}
$$

From the Kolmogorov definition of conditional probability, $p(A \cap B)=p(B) p(A \mid B)$

$$
\begin{aligned}
r_{k}(\boldsymbol{Y}, \boldsymbol{J}, \boldsymbol{E}) & =\frac{p\left(\bigcap_{i \leq k-1} \boldsymbol{Y}_{i}\right)}{p\left(\bigcap_{i \leq k-1} \boldsymbol{J}_{i}\right)} \frac{p\left(\boldsymbol{Y}_{i} \mid \bigcap_{j \leq k-1} \boldsymbol{Y}_{j}\right)}{p\left(\boldsymbol{J}_{i} \mid \bigcap_{j \leq k-1} \boldsymbol{J}_{j}\right)} \\
& =r_{k-1}(\boldsymbol{Y}, \boldsymbol{J}, \boldsymbol{E}) \times R_{k}
\end{aligned}
$$

which completes the proof.

Scenario probabilities can also be calculated.

$p(\boldsymbol{Y} \cap \boldsymbol{E})=\prod_{i \leq n} p_{i}(\boldsymbol{Y})$ and $p(\boldsymbol{J} \cap \boldsymbol{E})=\prod_{i \leq n} p_{i}(\boldsymbol{J})$, then use $r(\boldsymbol{Y}, \boldsymbol{J}, \boldsymbol{E})=\frac{p(\boldsymbol{Y} \cap \boldsymbol{E})}{p(\boldsymbol{J} \cap \boldsymbol{E})}$

Where there are multiple alternative scenarios $\boldsymbol{Y}^{(j)}$ and/or $\boldsymbol{J}^{(j)}$ use,

$p\left(\boldsymbol{Y}^{(j)} \cap \boldsymbol{E}\right)=\prod_{i \leq n} p_{i}\left(\boldsymbol{Y}^{(j)}\right), p\left(\boldsymbol{J}^{(j)} \cap \boldsymbol{E}\right)=\prod_{i \leq n} p_{i}\left(\boldsymbol{J}^{(j)}\right)$, $r(\boldsymbol{Y}, \boldsymbol{J}, \boldsymbol{E})=\frac{\sum_{j} p\left(\boldsymbol{Y}^{(j)}\right)}{\sum_{j} p\left(\boldsymbol{J}^{(j)}\right)}$

In practice, the sums are expected to be dominated by one or two most likely scenarios, and so, it is not necessary to consider a large number of alternatives.

The $p(\boldsymbol{H} \cap \boldsymbol{E})$ are prior probabilities of the scenario or subscenario $\boldsymbol{H}$ in combination with the evidence $\boldsymbol{E}$. To get the posterior probability for each scenario conditional on the evidence, it is necessary to divide by the prior probability of the evidence $p(\boldsymbol{E}) \cdot p(\boldsymbol{E})$ can be estimated by summing over the scenarios if they are known to include all likely possibilities.

$$
\begin{gathered}
p(\boldsymbol{E}) \approx \sum_{j} p\left(\boldsymbol{Y}^{(j)}\right)+\sum_{j} p\left(\boldsymbol{J}^{(j)}\right) \\
p(\boldsymbol{Y} \mid \boldsymbol{E})=\frac{p(\boldsymbol{Y} \cap \boldsymbol{E})}{p(\boldsymbol{E})} \approx \frac{r(\boldsymbol{Y}, \boldsymbol{J}, \boldsymbol{E})}{1+r(\boldsymbol{Y}, \boldsymbol{J}, \boldsymbol{E})}
\end{gathered}
$$

For person $C$ who had a possible identity switch or age change in the year $T_{S}$ and then lived until the year $T_{D}$, part of the evidence $\boldsymbol{E}_{D}$ including the death certificate shows that $C$ lived between these times. This evidence may not be the last evidence chronologically, for example, there may be DNA tests performed after death. It can be conveniently placed in the sequence just after the switch event. If evidence and 
scenario facts before are $\boldsymbol{Y}_{S}$ and $\boldsymbol{J}_{S}$, then the likelihood ratio for $\boldsymbol{E}_{D}$ (the mortality ratio) can be written as follows:

$$
R_{m}=\frac{p\left(\boldsymbol{E}_{D} \mid \boldsymbol{Y}_{S}\right)}{p\left(\boldsymbol{E}_{D} \mid \boldsymbol{J}_{S}\right)}
$$

Each scenario and evidence in the conditional part give the age of $C$ in the years $T_{S}$ and $T_{D}$. Mortality tables provide data $m_{C}(A)$ for the probability that a person $C$ who is alive at age $A-1$ will die at age $A$ years. The probability at birth for $C$ surviving to an age $A$ or greater is then given by the following:

$$
s_{C}(A)=\prod_{a=0}^{A-1}\left(1-m_{C}(a)\right)
$$

In the case of Mme Calment, an analysis of the longevity of her ancestors suggests that due to her privileged background, her mortality is most accurately represented by data for people living recently. ${ }^{4}$ Standard mortality tables for French women in 2014 (Ref. $^{8}$ ) are used to compute the mortality ratio. For ages $>105$ years where the mortality data are unreliable or not available, a mortality of 0.5 is assumed. ${ }^{4}$ If as some people believe, the true mortality is higher at extreme ages, then the mortality ratio will be higher favoring the switch scenario.

Let $J_{S}$ be the age of $C$ in the year $T_{S}$ according to the base scenario, and let $Y_{S}$ be the age of $\mathrm{C}$ in the year $T_{S}$ according to the switch scenario. Then the mortality ratio $R_{m}$ is given by the following:

$$
\begin{aligned}
R_{m} & =\frac{m_{C}\left(Y_{D}\right) \cdot s_{C}\left(Y_{D}\right) / s_{C}\left(Y_{S}\right)}{m_{C}\left(J_{D}\right) \cdot s_{C}\left(J_{D}\right) / s_{C}\left(J_{S}\right)} \\
& =\frac{m_{C}(99) \cdot s_{C}(99) / s_{C}(36)}{m_{C}(122) \cdot s_{C}(122) / s_{C}(59)}=2,000,000
\end{aligned}
$$

A factor for the probability of death in the year $T_{S}$ is not included in this ratio because deaths from specific medical conditions such as TB are not predicted by standard mortality tables. TB is believed to have been the underlying cause of death in 1934 in Calment's case.

As a sensitivity test, the calculation was repeated with cohort data for the birth years of Jeanne and Yvonne instead of the 2014 data. The results were similar, demonstrating that the number is robust. Note also that there is some medical evidence that Mme Calment who survived beyond $T_{S}=1934$ also suffered an episode of TB before that time. This illness could affect her mortality rates in an unquantifiable way, ${ }^{9}$ but we assume that it affected her probability of death at any age by a factor that depends only on the length of time since the illness. In this case, the mortality ratio is unaffected.

\section{What We Know: A Summary of Evidence}

It is beyond the scope of this report to present the full wealth of new evidence that has come to light concerning the life of Mme Calment. This includes photographs, letters, financial documents, medical records, and the testimony of relatives. For the purposes of our analysis, a summary of our conclusions drawn from this evidence is as follows:
1875-1898: Jeanne Calment was born on February 21, 1875. She married her double second cousin Fernand Calment on April 8, 1896, and gave birth to her daughter Yvonne on January 19, 1898, in Arles. Yvonne was her only child.

1922-1925: Yvonne became engaged to Joseph Billot, perhaps before he parted for a tour of duty in Morocco with the French army in 1923. Around this time, Yvonne appears to have suffered from an infection, which affected her face especially above her eyes and on her nose. This accounts for visible swelling that persisted in several of her subsequent photographs (image ref $P Y C 5^{10}$ ). Her right eye was affected more than the left. Although we do not know for sure, it is possible that this infection was a form of extrapulmonary TB. Mme Calment reported that Yvonne's pulmonary illness broke out after her son was born in 1926, so if it was TB they probably did not recognize her eye problem as TB at this time. ${ }^{11}$

1926-1927: On February 3, 1926, Yvonne married Joseph and on December 23, 1926, she gave birth to a son named Freddy. Jeanne was the godmother at the baptism on January 9, 1927.

1928: On June 10, 1928, Joseph took leave without pay from the army. The reasons for this that he gave to his superiors were his interests in Arles and the health of his wife. ${ }^{12}$ His "interests" could have been his financial affairs, including his family interest in the Calment drapery business. It was 10 years before he returned to serve in the war effort. This evidence suggests that Yvonne had latent TB that had returned after the birth of Freddy. It may have developed into more serious pulmonary TB or tuberculous pleurisy. Latent TB may progress in this way after pregnancy due to a weakened postpartum immune system. ${ }^{13}$

1929: Less than a year later on May 14, 1929, Yvonne was pictured with Joseph at the wedding of her brother-in-law Paul Billot (image ref PYCJB $1^{10}$ ). Another picture shows her participating in the Arlesian costume festival held in the grounds of St. Trophime (image ref PYCG5 ${ }^{10}$ ). Investigations by François Robin-Champigneul based on comparisons with other historical pictures of the location suggest that this was taken in 1928-1930. Yvonne showed no visible signs of ill health in either picture. She may not have been completely well, but she would be unlikely to attend a wedding or a festival if she was suffering from severe pulmonary TB at that time. No pictures of Jeanne or Fernand at the wedding or festival are known.

We know from the medical thesis of Dr. Levraud that Mme Calment had pleural sequelae visible on her chest $\mathrm{X}$-ray at the age of 111 years. ${ }^{14}$ This evidence indicates that she had a serious pleural infection of some sort previously in her life. Given the role that TB plays in their story, we take this as strong evidence that both Yvonne and Jeanne probably suffered from TB. Jeanne could have contracted it from Yvonne around 1928, for example. This conclusion is independent of whether an identity switch took place or not.

1931-1932: Yvonne was photographed in Leysin posing with a parasol in August 1931 (image ref PYCL1 ${ }^{15}$ ). From landmarks seen in the photograph, we know that she was at the Belvedere sanatorium which specialized in the treatment of pulmonary TB. The sanatorium had been reopened late in 1930 after renovation. Although Yvonne does not 
appear to be suffering from the kind of weight loss associated with pulmonary TB, the swelling over her eyes is still visible and may indicate an ongoing extrapulmonary bout of the disease.

Another photo that appears to have been taken around the same time shows both Yvonne and Jeanne (image ref PYCJC2 ${ }^{15}$ ). Yvonne's hairstyle matches the Leysin photo. It is not certain where it was taken, but it could be in Leysin or a sanatorium elsewhere. The flowers surrounded by foliage in front of Jeanne could be carline thistle (carlina acaulis) - a stemless alpine plant with white or yellow petals native to Switzerland and used as a herbal remedy for chest infections, including TB. ${ }^{16}$ Their presence was noted in the pastures just above the town near the sanatoriums. ${ }^{17}$ Jeanne and Yvonne are in formal dress as was usual for dinner at the Belvedere. ${ }^{18}$ The flowers and a wrapped gift in front of Jeanne could be a visiting gift.

In 1931, a census return indicates that Jeanne (misnamed as Maria), Fernand, Maria, Joseph, Freddy, and two servants are living together in Arles. Yvonne is not included. It is not necessary to detail here the many reasons why census returns in any country at any time can be unreliable. Even if this record is accurate, it only records the circumstances at one specific time and is comfortably consistent with any of the scenarios considered. It will not therefore influence the likelihood ratios and does not need to be considered further.

1933: The family acquired a villa in Paradou outside Arles. According to the testimony of Mme Calment, Yvonne returned from hospital to their home in Arles in December 1933 suffering from pleurisy. Joseph's military leave was extended for a second term of 5 years. It is not known what reasons he may have given for the extension.

There is a testimony from this time written by Pierre Maxence, son of an employee at the Calment drapery shop in Arles. ${ }^{19}$ He was 6 or 7 years old at this time and recalled playing in the shop with Freddy. He made the interesting statement that while Fernand was often in the shop, his wife only came there very rarely. At his age, he would have few memories of earlier times. He would not necessarily be able to distinguish between Jeanne and Yvonne at this time if they had been absent for some time and a switch had already taken place. Interesting as his story is, this testimony is comfortably compatible with any of the scenarios, switch or nonswitch, described below. It therefore does not affect the likelihood ratios significantly.

1934: On January 20, 1934, the family held a funeral for Yvonne. The event was announced in the press and many people witnessed the funeral cortège, but it is not known if an open-casque wake was held.

1934-1997: Mme Calment lived on with the rest of the family. She spent at least some of her time at the villa in Paradou. From 1963, after Fernand, Joseph, and Freddy died, she lived mostly at the apartment in Arles, attending church and visiting the family grave regularly. She socialized with family and friends and none of them had any reason to suspect that she was not Jeanne Calment. In 1985 she moved into the Maison du Lac retirement home. Shortly after, she became internationally famous for her unusual longevity. If she was not telling the truth about her identity then she played the role of Jeanne perfectly for at least 63 years until she died on August 4, 1997.

\section{The Base Scenario}

The base scenario $\boldsymbol{J}$ takes the evidence at face value. Yvonne died of pleurisy in 1934 following a long fight against TB. It is a disease that can subside and reappear in different forms where treatment with antibiotics is not available, as was the case at that time. It is possible in this scenario that Jeanne Calment also suffered from TB and that this accounts for the pleural sequelae seen on her X-ray later in life. Other conditions could also account for it, but it would have to be something serious to leave a trace after such a long time. However, most proponents of the nonswitch scenario do not accept that Jeanne contracted TB, so it will be assumed for our Bayesian analysis that she did not.

We know that Yvonne was ill enough in 1928 for Joseph to take unpaid leave from the army, but we also have photographic evidence that from 1929 to about 1931 she was in good enough health to attend a wedding and a costume festival and to be pictured in Leysin looking quite healthy. The base scenario therefore requires that she had a very serious relapse of ill health after 1931. It is difficult to reconcile her apparent good health in the Leysin picture with her being treated there for pulmonary $\mathrm{TB}$, but that is what the base scenario requires. In this scenario, the extension of Joseph's leave in 1933 is accounted for due to his wife's worsening health at that time.

According to this version of Jeanne Calment's story, her life was extraordinary in a number of ways. Her bourgeoisie background, the tragic loss of members of her close family, and of course her unique record of longevity.

If this base scenario is correct, then the longevity validation stands.

\section{Switch Scenarios}

The switch scenarios focus on events between 1927 and 1934. Before 1927, the course of events was the same as described for the base scenario, and after 1934, the difference is that Yvonne survived instead of Jeanne.

Assuming that the identity switch did take place, there are still many important details we do not know about the events of these years. When and where did the switch happen? What was the motive? Who knew? Where did they live or travel? Who looked after Freddy? Recall that evidence that these scenarios are correct is not required for the Bayesian inference methodology. To rebut the validation, it is enough to find a hypothetical sequence of events and facts that have a sufficiently high probability.

It is not possible to cover all possibilities so just two representative scenarios are considered. The motivation, location, and time for the identity switch in each case are different. The first scenario is an updated version of that proposed originally by Nikolay Zak where financial gain was the motive for an identity switch. ${ }^{4}$ The second is inspired by new evidence which suggests that faked signatures and the need to evade being discovered could be the motivation. The reality may have been a mixture of these or some different course of events that we are not aware of.

\section{Original switch hypothesis scenario}

For the original switch hypothesis $\boldsymbol{Y}^{(1)}$ the following facts are assumed: 
1920-1928: Mme Calment reported that cataracts were discovered when she had a major episode of conjunctivitis at age 20 years. ${ }^{20}$ Under the switch hypothesis, this is assumed to correspond to the swelling seen around her eyes (image ref $\mathrm{PYC} 5^{10}$ ). We think this could be TB, but we do not assume she had TB until later. This may or may not have been correctly diagnosed at that time. After the birth of Freddy, Yvonne has pulmonary TB. This accounts for pleural sequelae observed on her chest X-rays in much later life. By 1929, she has recovered from the pulmonary condition but may still have latent TB.

1927-1930: Jeanne spends very little of her time in Arles after the baptism of Freddy. From this time, she would be at the farm in Saint Martin de Crau taking care of her elderly father Nicolas Calment until his death on January 28, 1931. Around 1930-1931 she becomes ill with TB, perhaps she had caught it in 1927 or 1928 while caring for Yvonne.

1931: Yvonne and Joseph take Jeanne to Leysin where she is admitted to the sanatorium for heliotherapy and the photographs are taken. Yvonne returns to Arles but mostly lives out of town for the next few years. In response to changing fashions, she lightens her hair using hydrogen peroxide and hair dyes. ${ }^{21}$ Because of this, she is mistaken for her mother by those who have not seen them for a few years. This is possible because Jeanne had looked young for her age at the time of her daughter's wedding. Yvonne's sister in-law Antoinette Flauder is reported to have said that despite being 51 years old, Jeanne Calment appeared very young and that "with no exaggeration," she could have been mistaken for the bride. ${ }^{22}$

Yvonne wants to prevent Jeanne's illness becoming known because TB is a stigmatized disease and there is a concern that knowledge of her condition could bring shame on the family and affect their business. She therefore allows people to believe she is Jeanne.

1933-1934: Jeanne's condition degenerates to pleurisy so she returns home from Leysin to Arles. The family will be liable for inheritance taxes and notary fees if Jeanne dies. The retail business has suffered during the economic depression. We cannot rule out the possibility that Yvonne has a life insurance policy. Louis Ginoux, a witness at Yvonne's wedding, was an insurance agent. If Yvonne has insurance and Jeanne does not, then this is further motive for the switch. For these reasons, Yvonne and close family members who knew about Jeanne's condition decide to make an identity switch so that Yvonne appears to have died rather than Jeanne. The funeral is held with a closed coffin to prevent infection and Yvonne hides her face behind a veil. In this way, very few people are aware of the switch despite a large attendance at the funeral. In this scenario, the reasons for the extension of Joseph's leave in 1933 would more likely relate to his financial interests.

1934-1945: In the years that follow, Yvonne avoids being seen in Arles by anyone who might recognize her. She lives mostly at the villa in Paradou and enjoys hunting rabbits with her pet Jack Russell. Freddy goes to school in Arles. He is taken to school by car driven by Joseph so that Yvonne is not seen. In time she ages and is no longer in danger of being recognized as Yvonne. Graying hair (or hair dye) and the effects of the earlier episode of TB may have helped make her look older.

\section{New switch scenario}

One reason that the original switch hypothesis may have a low probability is that the motive is financial. Faking your own death and assuming your mother's identity is a drastic action. To do this for money alone is unlikely unless it could save them from a very difficult financial position. The probability may be higher if there was a more compelling motive. With this in mind, a new alternative switch scenario $\boldsymbol{Y}^{(2)}$ is proposed. In this version of events, the financial motive is less important. Instead, Yvonne creates an innocent fiction that grows into something more culpable until she finds herself in a circumstance where the identity switch is her only safe option to avoid a scandal. ${ }^{23}$

Events in the new switch scenario are similar to the original version except that the identity switch begins in Leysin. This could happen if both Yvonne and Jeanne are admitted to the sanatorium at the same time. Yvonne may have had premature gray hair, a genetic trait also known as canities or achromotrichia. In the parasol picture, her hair seems unnaturally dark and shiny. This is a common effect of using hair dyes of the time (such as black henna), which were marketed for covering gray hair. At the sanatorium, she may have stopped using the hair coloring with the result that she appeared older and was confused with Jeanne. A photograph, taken in 1937 in Versailles, shows Mme Calment with a full head of light colored hair (image ref $\mathrm{PMCl}^{24}$ ). Either it was gray or she lightened her hair with cosmetic products. In the picture of Jeanne and Yvonne together (image ref PYCJC $1^{15}$ ) neither shows signs of gray hair and this was probably around 1931. Even in a nonswitch scenario, we would therefore have to conclude that Jeanne was using hair dye.

Yvonne's condition improved so she returned home. There she pretended to be Jeanne to cover for her mother's illness. Explaining Yvonne's absence was easier because she had previously been traveling with Joseph. She had fewer obligations than Jeanne. It was a small invention to hide the shame the family felt because of the stigma of TB. To minimize the risks, she would restrict her contacts to those who would not distinguish Yvonne from Jeanne. Joseph could also keep away from Arles or avoid contacts between 1932 and 1933.

France has always been renowned for its unforgiving and burdensome bureaucracy and it is likely that Jeanne was required to sign various documents in Arles in connection with her finances or property transactions. As her husband, Fernand could sign some documents on her behalf, but that would raise questions. The family could not let it be known that she was being treated for TB $500 \mathrm{~km}$ away in Leysin. Yvonne would have to sign the documents with a forged signature. Some signatures may have required ID for authentication. Yvonne obtained an ID card in Jeanne's name using Jeanne's passport brought back from Leysin to validate it. The new ID card had an old photo of Jeanne but a fake signature written by Yvonne. With this as ID, Yvonne was able to forge signatures in Jeanne's place. Although these events are unverified, a copy of such an ID card exists. The details on the card may have been copied from a passport. Her eye color is down as black, when Mme Calment's actual eye color was green.

In this scenario, the main reason Joseph would give for the extension of his military leave is likely to be the 
continuing ill health of his wife since they would be pretending that it is she who is still seriously ill.

Yvonne had mostly recovered by this time and she assumed that Jeanne would too, so she did not expect to gain from the practice of faking signatures. It just helped to keep Jeanne's condition secret. They assumed that the identity switch could be quietly reversed after Jeanne recovered. However, Jeanne's TB suddenly degenerated to pleurisy and her condition rapidly declined at the end of 1933. She had to be brought back to Arles. She may have returned on Yvonne's passport if they had switched in Leysin.

At this moment, Yvonne was masquerading as Jeanne. Notaries who had seen her sign documents in Jeanne's name would know Yvonne as Jeanne. This put Yvonne and her close family in an awkward position. There was no time for Jeanne to have become suddenly ill and she would look different from Yvonne who was presently known as Jeanne. They could not admit that the person who had returned ill was the real Jeanne because then the ongoing deception and faked signatures would become known. It would mean a scandal if discovered so Yvonne could not revert to her real identity. This left the family with no option. They now had to tell everyone including doctors who examined her that Jeanne was Yvonne, and when Jeanne died they had to say that it was Yvonne who had succumbed to pleurisy. This would readily be accepted by people who had known about Yvonne's earlier bout of TB before she switched identities. The family was then never able to reverse the identity switch because Jeanne's death had been recorded as that of Yvonne in front of all the witnesses at the funeral.

Yvonne's prematurely graying hair and aging appearance due to the earlier bout of TB always helped her appear older so that almost nobody ever suspected what had happened. Extrapulmonary TB can also cause swelling of the parotid gland so that cheeks become flabbier afterward. Cutaneous TB would account for older looking skin. After a few years, the risk of being recognized as Yvonne diminished. Despite the effects on her appearance, the TB would not necessarily affect her life expectancy.

The identity deception grew to global proportions in 1986 when Mme Calment became famous for her longevity.

\section{Further Evidence}

There are many items of circumstantial evidence in favor of the switch hypothesis that have been discussed. ${ }^{4,5}$ For this Bayesian analysis, we use just two pieces of further evidence that we consider substantive.

\section{Eye trauma}

There are two pictures of Mme Calment taken in about 1943-1945 (right in image ref $\mathrm{PMCC}^{24}$ ) The patches of color in her hair match in both these later photographs, and so, we are confident that they are the same person. If these are compared with earlier pictures of Jeanne and Yvonne, it is very hard to tell which one she is. Some will say she looks like Jeanne and other like Yvonne. It seems to depend on the expression on her face, or the angle and lighting. When together in a room at the same time, they would have been easy enough to tell apart, but over encounters or photographs separated by years they would have been harder to tell apart.
It is this striking similarity between mother and daughter despite their age difference that made a switch possible.

In many of her pictures, including the Leysin photographs (image refs PYCL1, PYCJC2), Yvonne is seen to have some swelling above her eyes, especially her right eye. We believe this is due to some serious infection such as TB. It is not seen on her earliest photographs and so it is not hereditary. Matching eye trauma is visible in the lower photo of Mme Calment taken around 1940-1945. Note that there are some different copies of this picture where the swelling is less obvious and its presence is disputed, but in our opinion it is still there.

\section{Signatures}

Figure 2 shows 15 samples of signatures written by Jeanne Calment or Mme Calment between 1896 and 1992.

The signatures show an evolution over time, as expected, but between 1927 and the 1930s a more unnatural change occurs. The initial " $\mathrm{J}$ " changes shape at the top from pointed to rounded making the loop more symmetrical, while the final " $t$ " loses its underline tail. While signatures can begin to show changes over periods of only 6 months, changes of this type indicate a difference of writing style that is not normally seen in someone in their 50s. For the next 40 years after 1930, the signature again changes only by natural forms of evolution.

The 1930s signature, which first shows the change, is from an ID card. It is possible that Yvonne forged the



FIG. 1. Space $\Omega$ of possible histories. 


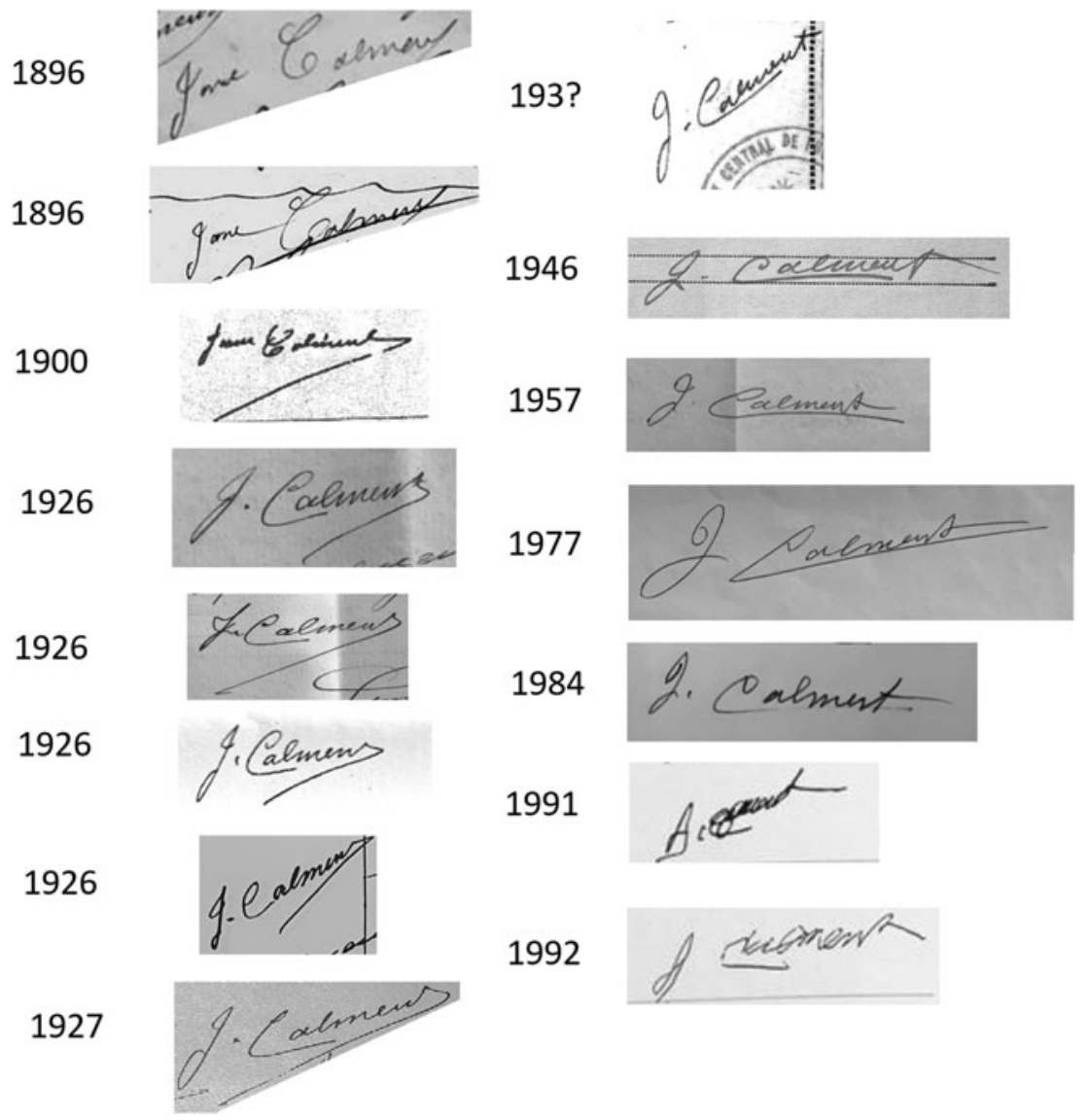

FIG. 2. Jeanne Calment signatures.

signature on this card in her mother's name so that she could use it to validate other forged signatures.

\section{Overall Likelihood Ratio}

To calculate $r(\boldsymbol{J}, \boldsymbol{Y}, \boldsymbol{E})$ the overall likelihood ratio for switch versus no-switch, the Bayesian inference lemma is used. The evidence consists of the 33 official documents found for the original validation, plus photographs, letters, signature analysis, and so on. These are to be combined with the hypothetical events in each scenario variant to form the sequences of facts $\boldsymbol{J}_{i}$ and $\boldsymbol{Y}_{i}$ so that the ratios $R_{i}=\frac{p\left(\boldsymbol{Y}_{i} \mid \bigcap_{j<i} \boldsymbol{Y}_{j}\right)}{p\left(\boldsymbol{J}_{i} \mid \bigcap_{j<i} \boldsymbol{J}_{j}\right)}$ can be calculated.

If events and evidence from before 1931 are taken first, then the ratios for these facts are simply equal to one because there are no differences between the switch and nonswitch scenarios up to this time. These facts remain relevant in the conditional part of later probabilities but do not contribute directly to the likelihood ratio.

The documentary evidence that recorded Yvonne's death, rather than Jeanne's, plays against the probability of the switch having taken place. However, once one piece of evidence is included, any subsequent documentary evidence is conditional and contributes much smaller factors to the likelihood ratio. Once it is accepted that the original documents were wrong but successfully recorded, the later records are likely to follow suit. Subsequent evidence does, however, show that she was not later caught and the probability for this has been included.

Some likelihood ratios such as mortalities can be calculated from known statistics. In other cases they cannot. An individual can estimate a likelihood ratio for each fact according to the "Dutch book argument." That is by considering what breakeven odds they would accept in circumstances where betting for or against the outcome was possible. ${ }^{25,26}$ This is both subjective and hypothetical, and so, the numbers used vary from person to person.

Overall we estimate the likelihood ratio for the switch scenarios to be as follows:

$$
\begin{aligned}
r(\boldsymbol{J}, \boldsymbol{Y}, \boldsymbol{E}) & =\frac{p\left(\boldsymbol{Y}^{(1)} \cap \boldsymbol{E}\right)+p\left(\boldsymbol{Y}^{(2)} \cap \boldsymbol{E}\right)}{p(\boldsymbol{J} \cap \boldsymbol{E})} \\
& =\frac{8.0 \times 10^{-13}+8.0 \times 10^{-13}}{1.6 \times 10^{-16}}=10,000
\end{aligned}
$$

See Table 1 below for details. In combination, this implies a probability for switch of

$$
p(\boldsymbol{Y})=\frac{r(\boldsymbol{J}, \boldsymbol{Y}, \boldsymbol{E})}{1+r(\boldsymbol{J}, \boldsymbol{Y}, \boldsymbol{E})}=99.99 \%
$$

We accept that some of the probability estimates we have used are subjective. We do not claim that it is possible to conclude that the switch hypothesis is almost certainly 

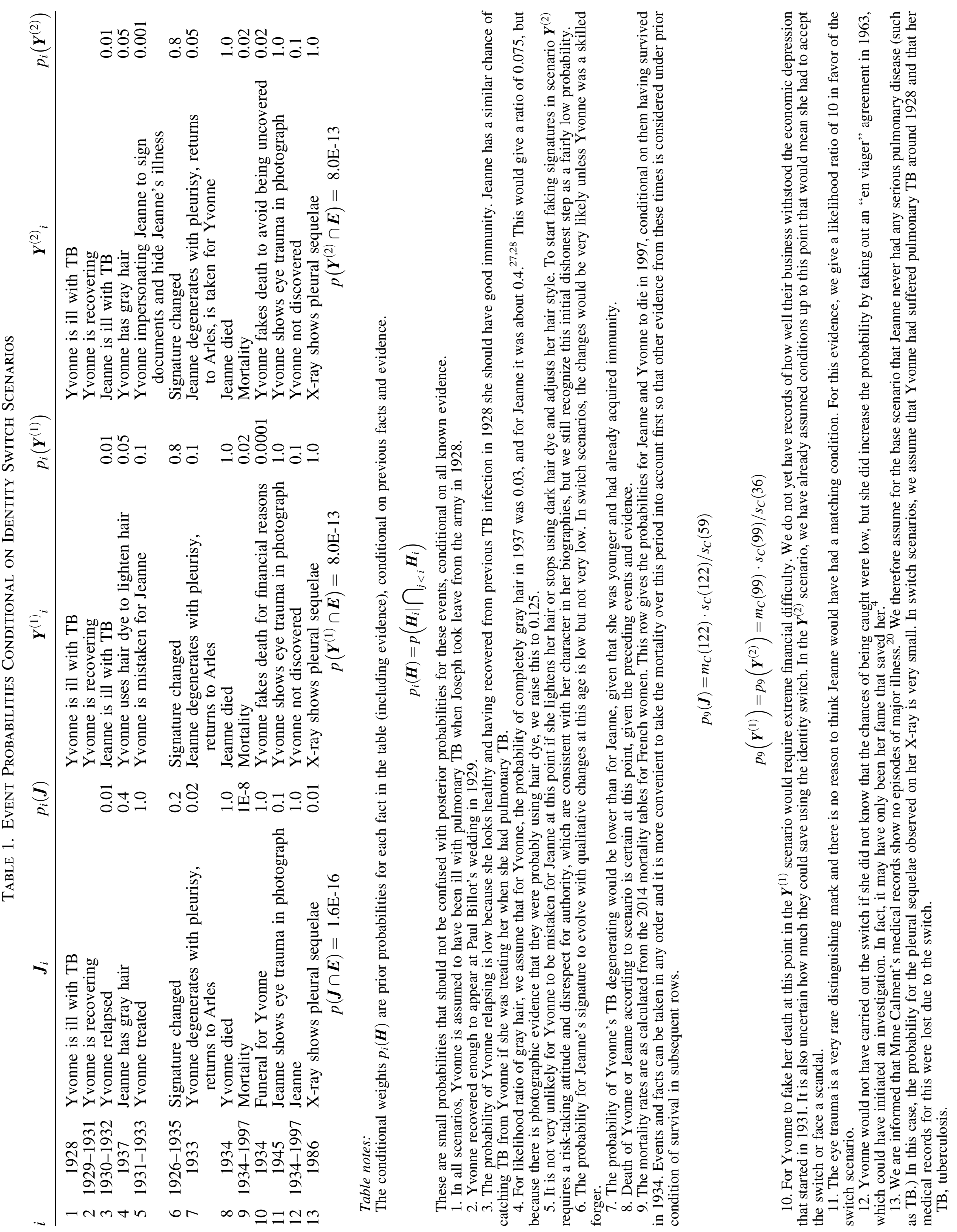
proven, but we do believe that there is genuine reason to doubt the base scenario and that the longevity validation has been called into question, and to seek objective DNA tests to resolve the uncertainty.

As an alternative base scenario, the case where Jeanne also had pleural TB at some time before 1934 could be considered. For this scenario, the 0.01 factor in row 13 would be taken out, but instead a factor of 0.01 would be required for Jeanne contracting the TB. It would also be necessary to explain why this was not shown on her medical record, which might require an extra factor of 0.1 . The probability for this base scenario would therefore be lower by a factor of 10 .

\section{Response to Counterclaims}

It is necessary to address some commonly made claims about why the switch hypothesis is impossible or much less likely than presented.

"Arles is a small town where everyone would know the affairs of the Calment family, so they all had to hide the truth."-We think that if the switch hypothesis is true, Jeanne was rarely seen in Arles between 1927 and 1935, while Yvonne was absent from 1930 to 1931. When Yvonne was seen after about 1932, her gray hair meant that she was mistaken for Jeanne by anyone who did not know her well. There is no testimonial evidence to contradict this. For example, information saying that Jeanne attended church in Arles every Sunday is based on events after 1960. Only a few close family members had to be aware of the switch. The testimony of Pierre Maxence says that Mme Calment was rarely seen in the shop. ${ }^{19}$ If he had described her presence in detail, it might have counted against the switch scenario, but as it stands it is consistent with both switch and nonswitch.

"The funeral was very public. There was a long list of family members who confirmed the death of Yvonne on invitations sent to friends and in the press."-Most of the family members listed on the funeral announcements lived outside Arles and may not have had time to attend the funeral. They may not have been aware of the switch. These announcements are prepared by the immediate family of the deceased at short notice and follow standardized protocols. They do not require everyone listed to verify the circumstances. It is claimed that witnesses made it hard for Yvonne and Jeanne to switch. In fact, the opposite is true. Witnesses and distant relatives had been led to believe that Yvonne was Jeanne and/or that it was Yvonne, not Jeanne, who was seriously ill, so when Jeanne returned in ill health the family had to pretend that she was Yvonne. The witnesses to this then made it impossible to revert the switch.

"The body would have been seen at the funeral by many people who would have recognized Jeanne."-Although it was traditional to hold an open-casqued wake, there is no direct documentary evidence that this was the case here. It may have been advised to close the coffin due to the risk of infection. Even if the body was visible, nobody had seen Jeanne since 1927. She had looked young and had a strong resemblance to her daughter. Yvonne had not been seen since 1930 and also changed appearance. Even a doctor or priest who may have seen her before or after death could not be counted on to identify her.
"Freddy would have recognized his own mother in place of Jeanne and was too young to keep a secret." - Very little is known about who took care of Freddy during his early years up to the age of 7 in 1934. For much of that time, both Yvonne and Jeanne would have been away from Arles, either traveling or being treated in sanatoriums. After 1934, Freddy was instructed to treat Mme Calment as his grandmother. Young children tend to accept what they are told by family. If there had indeed been a switch, then this protected him from having to support the falsehood. There is no reason to think he would ever have suspected a switch.

"The family has testified that Mme Calment was well known to them and never gave any indication that she was Yvonne. Photographs, letters, and other documents have been produced to support their case." - There is no reason to doubt the integrity of living relatives. None of them has recollections of Jeanne from before 1934. None of the evidence or testimony provided by anyone is inconsistent with the switch hypothesis assuming that Mme Calment kept it secret from all but a few people and that she looked older due to canities and the effects of TB.

"The validation of longevity for Mme Calment is proven with more than 30 official documents by expert gerontologists who interviewed Mme Calment and knew her well."'This case will not be settled by the authority of experts, only by the evidence. The quantity of official documents does not help refute the switch hypothesis since each record depends on previous records for verification of identity. In Bayesian analysis, the likelihood ratio for each item of evidence uses probabilities conditional on previous evidence.

"If the mother/daughter identity switch is as probable as claimed, there would be many other examples. Where are they?"-It is possible that other mother/daughter switches have taken place, but were undiscovered because they did not result in an exceptional claim of longevity, but it is also possible that nothing remotely similar has ever happened elsewhere. The circumstances depicted in the switch scenarios are unique and the conditional probabilities for the switch-related parts of the scenarios are calculated to be one in 2.5 billion. There is therefore no reason to expect anything similar to have occurred elsewhere. Many combinations of unusual events happen to people every day. Suppose a coin was tossed 30 times and the sequence of heads and tails was noted down. The prior probability for this sequence is $<1$ in a billion yet it happened and the recorded evidence supports it. Similarly, it is not unlikely that a highly unusual sequence of events befell the Calment family.

"The Bayesian analysis says that Jeanne Calment's longevity is improbable, but it can be shown using extreme value theory that improbable events do happen. Jeanne Calment's age record is like Bob Beamon's long jump record, a statistical outlier that stood for decades." -Extreme value theory can only be used when the probability distribution is known or can be extrapolated. Different models for mortality rates at extreme ages give very different predictions and the true rates are not known. In this Bayesian analysis, we use a mortality plateau model, which is the most conservative consistent with demographic data. The conclusion that her record is improbable is not based on whether 122 years is an unlikely world record for longevity. It is based on a comparison of the probability for Mme 
Calment's age as an individual, with other possible scenarios for her own life story.

"There is no evidence to support the switch hypothesis. It is just unfounded speculation."-An equally correct statement is that there is insufficient evidence to support the longevity validation of Mme Calment. Many details of their situation in nonswitch scenarios as described by advocates are also unfounded speculation. It is not necessary to prove every detail of the switch hypothesis to cast doubt on the base scenario. In fact, there is now a considerable amount of evidence in favor of the switch. Despite many opportunities, no conclusive evidence has been produced to refute it. We do not claim that the switch theory is proven. We only ask that DNA evidence be used to settle the matter.

"The researchers who promote the pro-switch hypothesis have not even been to Arles. They cannot understand the life of Jeanne Calment."-It is 2019. Google provides instant street views of Arles, and relevant details of the region are easily found online. All the necessary information has been shared on the Internet and in numerous books written about Jeanne Calment. For example, the locations of many of the photographs used as evidence can be confirmed using Google views and old postcards found online. Visiting Arles would not provide any new insight. In fact, much missing evidence is more likely to be elsewhere. Furthermore, we have support for the switch hypothesis from some people who have lived in Arles. They know very well the town, its people, and the history of Jeanne Calment.

"The Calment household servants would have noticed the switch and let everybody know. For example, the cook Louise Gauzargues appears on both the 1931 and 1936 census living at the Calment address."-Only a very small number of servants are likely to have been close enough to the family for long enough to have observed the switch over the period of years when Jeanne and Yvonne could have been largely absent. However, it is likely that some such as Gauzargues would have known. Servants are expected to be discrete and their loyalty or the risk to their own reputation may have prevented them from talking. It is also possible that they did talk but were not believed. Indeed there was a report from Laure Meusy-Defousset, former head of the Maison du Lac, that a nurse at Mme Calment's retirement home whose grandmother worked in the Calment household had claimed that Yvonne had taken the place of Jeanne. ${ }^{29}$

"Family photographs of Jeanne Calment taken between 1943 and 1963 show that she looked much older than Yvonne would have been at this time. Furthermore, a testimony of Didier David who saw Jeanne Calment during the Second World War said that she appeared as 'une grandmere toute menue, de noir vetue, tres alerte et vive. ${ }^{30}$ Later, the notary Raffray famously signed an en viager deal believing her to be in her 90s. This would not have been possible if she was really 23 years younger."-Age estimations taken from appearance are often highly inaccurate. In the case of Yvonne, according to the switch scenario we believe that she had premature gray hair (canities), which she did not hide with hair dye after the switch. The longterm effects of TB could also have affected the appearance of her face and skin, giving her hooded eyelids (dermatochalasis), a drooping nose (nasal ptosis), and a swollen parotid gland in her cheeks. Given these conditions, it is not surprising that Didier David who was a young teenager at the time judged Mme Calment to look like a little old lady when he saw her white hair and small stature, despite her being alert and active. The photographs from 1940s to 1960s, which show her with white hair, are also consistent with the age of Yvonne at those times if we allow for these features of her appearance. Raffray could also have been fooled for the same reasons. Aside from her physical appearance, her actual health and fitness were reported to be more like that of someone 20 years younger.

"Jeanne Calment recounted many stories from her youth with details that Yvonne could not have known, such as the names of her teachers and a dressmaker."-Neither Jeanne nor Yvonne ever worked. They had plenty of time together and had common interests. It is entirely likely that Jeanne told Yvonne many details of her early life. Her teachers would have been well known in the community and some may have taught two or even three generations of the same families. Fernand was a vice president of the association of former pupils, so teachers and former teachers would be known to the Calments. Yvonne could have known many obscure details of her mother's life.

It is not clear how well Madame Calment's answers were really checked, or even who the teachers she is said to have named were. Tapes and full transcripts of her interviews with the validators have not been released, and so, it is impossible to know how much prompting and repetition may have been used. In fact, Mme Calment was often evasive in answering questions and confused her husband with her father. ${ }^{4}$ Many of the stories she told could have been the experiences of Yvonne rather than Jeanne. She was also interviewed many times by different people, including biographers and historians who met her long before the validation interviews began. They would return to the same questions so she had opportunity to adapt her answers and learn from what she was told. Her testimony warrants further analysis if original tapes are released, but it does not currently provide strong proof or disproof of her longevity.

\section{DNA Tests}

Although our calculation gives a $99.99 \%$ chance of an identity switch in the case of Mme Calment, we acknowledge the subjective nature of this result. We expect that some other investigators will not agree with our figures. An objective test is therefore needed to settle the question of Mme Calment's longevity validation. This is possible using DNA sequencing.

We know that Jeanne Calment and Fernand Calment were double second cousins. We have verified this ourselves by searching through all the relevant acts of birth, marriage, and deaths. We find the vital details to be unambiguous unless an earlier falsification took place. This means that Yvonne was the product of in-breeding between her parents with 5th degree consanguinity. This would result in detectable autozygosity in her DNA characterized by long runs of homozygous genes. On average, one in 32 allele pairs in her genome would be inherited from the same common ancestor. Normal levels of recombination in autosomes mean that these homozygous genes occur in long runs of homozygosity $(\mathrm{ROH})$, which can be detected and counted once the genome has been fully sequenced. For the level of consanguinity between Yvonne's parents, the actual count 
should be around $2 \%$ to $5 \%$ of the genome. DNA tests now in use can distinguish counts as low as $1 \%$ from background.

To complete the test, a number of laboratories should independently measure the fractional $\mathrm{ROH}$ from single nucleotide polymorphisms data for all the autosomes with a range of threshold sizes, for example, 1 and $8 \mathrm{Mb}$. Jeanne had no known common ancestors, so with a single test of Mme Calment's DNA, it should be possible to settle the question of her identity with a high degree of confidence. ${ }^{31}$

Other DNA tests could be used such as consanguinity with known living blood relatives of Yvonne who are not blood relatives of Jeanne. In particular, there are several living descendants of Emile Fassin, the former mayor of Arles who married Anne Felix, the sister of Yvonne's paternal grandmother. Some are second cousins once or twice removed from Yvonne Calment, but have no close blood relationship with Jeanne Calment. A test based on complete DNA sequencing can easily distinguish this relationship. This will be largely independent of the autozygosity test, which uses lines of ancestry passing through Yvonne's mother and her paternal grandfather.

Measurements of the epigenetic clock could also be attempted, but this may require a control study of other supercentenarians. $^{32}$

We understand that the Jean-Dausset foundation in Paris holds suitable biosamples from Mme Calment that could be used for this purpose if legal barriers can be removed. There is also a possibility that stamps on letters sent by Mme Calment to relatives could yield DNA samples. We understand that French law is strict on the use of DNA testing, but tests for scientific purposes are permitted. A legal decision may be required to determine if this use of the DNA is possible under the privacy conditions it was given. We urge the gerontological community of France to support organization for this to be carried out. According to Jeanne Calment's validator Jean-Marie Robine, she eagerly accepted brain imaging and blood tests to investigate her longevity. ${ }^{33-37}$

There may be ulterior interests in the DNA of Mme Calment from researchers working on the genetics of longevity. We understand that this leads to suspicions about motives for the DNA tests especially where financial gain may be possible. We, the authors of this article, wish to make it clear that we have no conflict of interest of this type. We are happy for any tests to be carried out by agents independent of any interested parties. This would be under conditions that would only reveal the derived numbers necessary for the tests described here to establish the identity of Mme Calment. There should be control samples tested blindly alongside Mme Calment's sample. The tests should be performed by at least three laboratories not linked to each other to ensure reproducibility. Although we would require the genome of Mme Calment to be fully sequenced, we do not require it to be published or made available to ourselves or anyone other than the independent agents. The possibility of scientific tests for other purposes is a separate matter and is not our interest.

We appeal to French gerontologists and the family of Mme Calment to support these DNA tests under conditions that they are free to propose. Only this will return the certainty that science and her family need to understand the life of this remarkable woman.

\section{Conclusion}

Our Bayesian analysis indicates that the balance of evidence gives reason to suspect that Jeanne Calment could be a case of identity switch. There is no single item of solid proof, but the trauma above Mme Calment's right eye in the picture of 1940s corresponds to the swelling seen in photographs of Yvonne from 1924 to 1930 . This is unlikely to be coincidental. The unnatural change in signature after 1927 is also substantive. It first appeared on a suspicious ID card with a photo of Jeanne Calment at least 20 years out of date. Mme Calment claimed vehemently that she had never been ill, yet the presence of pleural sequelae on her chest X-ray suggests otherwise. It seems that she was hiding her own historic infection with TB. Yvonne Calment was pictured at the Belvedere sanatorium in Leysin looking generally healthy, yet according to the validated scenario we must believe that she was there to be treated for pulmonary TB. It seems more likely to us that she had recovered and was there to accompany her sick mother instead. In combination with the improbability of reaching the age of 122 compared with 99, we calculate a likelihood ratio of 5000 to 1 in favor of switch for two different switch scenarios. This means that in our opinion, her validation should be rescinded until stronger proof of her longevity is provided.

There is a consistent hypothetical scenario for an identity switch between Jeanne Calment and her daughter Yvonne before Yvonne's recorded death in 1934. The evidence suggests that they both suffered from TB, but Yvonne recovered. The switch could have taken place a few years earlier while they were at the sanatorium in Leysin. Jeanne looked young and bore a close resemblance to Yvonne. Yvonne had premature gray hair or used cosmetic products to lighten her hair. She also suffered TB legions on her face that left trauma. This made her look older so that the switch became possible. Initially the switch was made to protect Jeanne and the family from the stigma of TB. The deception grew when Yvonne forged her mother's signature to cover for her absence. When Jeanne did not recover, it left Yvonne in a difficult position requiring her to continue with the charade to avoid a legal scandal. Financial gains were an additional incentive to continue with the switch, although we do not have full details of their financial position to confirm this.

If this is correct, then it is probable that very few people knew of the switch and the truth was not passed on to younger family members. Nobody noticed because Jeanne had been mostly absent from Arles for up to 7 years when she died and Yvonne's appearance changed.

It will be said that our scenarios are preposterous, unsupported by evidence, and that they could not have happened for various reasons. In fact, they are merely improbable, but less improbable that the relative likelihood of living to an age of 122 versus 99. Very little is known about the life and movements of the family in the years, months, and days before the funeral in 1934. When people say that these things could not have happened, they are making assumptions about events that are no better supported than what we propose is possible for an identity switch. No testimony or other evidence from people who knew the family before 1934 has contradicted it.

We believe the switch to be a credible scenario that casts the longevity validation into doubt. Despite the high likelihood 
ratio that we have calculated in favor of the switch, we do not claim that the matter is settled. Some elements of the computation are subjective. Instead, we propose DNA tests and we predict that the results will show that the identity switch did indeed take place. It is important to settle the matter from both a scientific and historic point of view. Fortunately, a biosample from Mme Calment exists in the possession of the JeanDausset foundation, which could be used to end the doubt. French law of bioethics is very strict on DNA testing. There are exceptions for scientific purposes, but it may still require a judicial decision to authorize it. The ability to make this test happen rests with the French gerontological community.

In the absence of DNA tests, there is other evidence that could be forthcoming and which might help answer whether or not the switch took place. In particular, medical evidence, including chest X-rays taken of Mme Calment in her late life, could tell us if she had an earlier episode of TB. This would require an assessment by a medical expert. It is possible that there are other archived records to be found, including documentation on the treatment of Jeanne or Yvonne in around the 1930s. There may be further military records concerning Joseph Billot's leave "en disponsibility" such as notes explaining why it was extended in 1933. More financial records of the Calment retail business or the personal affairs of the family would be useful. Any further documents containing the signature of Jeanne Calment between 1927 and 1940 would be of particular interest, including a complete copy of Jeanne Calment's 1930s ID card showing the full date. Census returns covering any of the properties owned by the extended Calment or Billot families between 1926 and 1936 could hold hidden clues. Finally, there are photographs of the family for which only low-resolution copies are available. It would be helpful if high-resolution copies could be made of all known family photographs before 1985 and any others that have not yet been made public. It would also help if copyrights could be explicitly put under free license for noncommercial uses such as scientific research.

If the Calment affair is not resolved now, time may provide the answer. The mortality ratio of 2 million to one for this case assumes that mortality rates plateau at a rate of 0.5 above 105 years. If the rate is higher as many people suspect, ${ }^{2}$ then her mortality ratio could be much higher and the probability for the switch increases with it. Although mortality rates could be higher, it would be premature of us to assume it. Global statistics may be overwhelmed by data errors, including possibly the case of Mme Calment. It would be a mistake to fall into a trap of biased selection and circular reasoning. However, as time goes by, vital records become more reliable, photographic records are more common, and DNA could be used to improve data quality. Within a few decades, data from more countries will be usable and the mortality curve will be more reliable at extreme ages. It may tell us that longevities in excess of 120 years are regular occurrences, in which case Mme Calment's identity will be less doubted, but it may also increase her mortality ratio by several orders of magnitude, beyond limits where anyone would reasonably be willing to accept her record.

In the wider field of longevity studies, we suspect that there could be other cases of possible misvalidation that could be further skewing estimates of mortality rates for supercentenarians. There is often potential for political pressure to validate cases of longevity. Prospective supercentenarians may sometimes be given the benefit of the doubt when evidence is minimal. We propose that a multitier certification procedure be introduced where the current validation criteria are counted as the lowest standard. DNA samples should be requested from all supercentenarians and their relatives to verify the expected levels of consanguinity. A public online database should be created for individuals who are willing to give permission. It should hold copies of all official documents, family photographs, testimony, results of DNA tests, and other evidence indicating any provenance. All available evidence should be subjected to a Bayesian inference assessment, which considers any plausible alternative scenarios. Many subjective estimates could be set by agreed standards to make the result more objective. If this test is passed, a higher tier of validation can be accredited. In time, this will improve confidence in our knowledge of mortality rates at extreme ages with corresponding benefits to medical practice for the elderly.

\section{Acknowledgments}

This work has benefited from the investigations of many people who have been searching for new evidence of Jeanne Calment's longevity, especially through the Facebook group "Contre Enquete Sur L'Enquete Jeanne Calment." In particular, we thank Michael Apicella, Aurélie Azam, Violette Bernard, Patricia Couturie, Yuri Deigin, Aubrey de Grey, Yann Martin, Valery Novoselov and Maguy Raspail, François Robin-Champigneul, and others who did not wish to be named. The inclusion of their names here does not imply that they endorse our findings in any way. François RobinChampigneul wishes it to be known that he opposes the identity switch hypothesis.

\section{Author Disclosure Statement}

No competing financial interests exist.

\section{Funding Information}

No funding was received.

\section{References}

1. Gavrilov LA, Gavrilova NS. Late-life mortality is underestimated because of data errors. PLoS Biol 2019;17: e3000148.

2. Newman SJ. Errors as a primary cause of late-life mortality deceleration and plateaus. PLoS Biol 2018;16:e2006776.

3. Robine JM, Allard M. Jeanne Calment: Validation of the duration of her life. In: Validation of Exceptional Longevity. Max-Planck-Gesellschaft, 2003. Available at https:// www.demogr.mpg.de/books/odense/6/09.htm (accessed June 24, 2019).

4. Zak N. Evidence that Jeanne Calment died in 1934, not 1997. Rejuvenation Res 2019;22:3-12.

5. Deigin Y. J'Accuse...! Why Jeanne Calment's 122-year old longevity record may be fake. December 10, 2018. Available at https://medium.com/@yurideigin/jaccuse-why122-year-longevity-record-may-be-fake-af87fc0c3133 (accessed June 24, 2019). 
6. Milova E. Valery Novoselov: Investigating Jeanne Calment's Longevity Record. New York, NY: Life Extension Advocacy Foundation, November 4, 2018.

7. Facebook. Available at https://www.facebook.com/groups/ 763398500702857 (accessed June 24, 2019).

8. The Human Mortality Database. Available at https://www .mortality.org (accessed June 24, 2019).

9. Hoger S, Lykens K, Beavers SF, Katz D, Miller TL. Longevity loss among cured tuberculosis patients and the potential value of prevention. Int J Tuberc Lung Dis 2014; 18:1347-1352.

10. Calment Wiki, fandom.com, Yvonne Calment. Available at https://calment.fandom.com/wiki/Yvonne_Calment (accessed June 24, 2019).

11. Allard M, Lebre V, Robine JM, Calment J. Jeanne Calment: From Van Gogh's Time to Ours, 122 Extraordinary Years. New York: W.H. Freeman and Company, 1998, p. 131.

12. Note found in military archives, Paris, for "Forges inspectorate of Paris [In French]." published on "The Jeanne Calment Riddle" France 2, March 21, 2019.

13. Kodadhala V, Gudeta A, Zerihun A, Lewis O, Ahmed S, Gajjala J, Thomas A. Postpartum tuberculosis: A diagnostic and therapeutic challenge. Case Rep Pulmonol 2016;2016: 3793941.

14. Levraud C. Dissertation for the competency of gerontology: Jeanne Calment 118 years, a longevity precedent [In French]. University Library of Medicine of Marseille. 1993.

15. Calment Wiki, fandom.com, Leysin. Available at https:// calment.fandom.com/wiki/Leysin (accessed June 24, 2019).

16. Fetrow CW, Avila JR. The Complete Guide to Herbal Medicines. New York, NY: Pocket Books, 2000, p. 112.

17. Mayor E. Study of the mycological flora around Leysin [In French]. Soc Vaud de Sci Nat Bul 1918;52:113-149.

18. Herter SB. No More Tiaras: (A Memoir of Eight Decades). Bloomington, IL: Xlibris, p. 147. 2011.

19. Maxence P. La Mainson Calment. Bulletin of the friends of old Arles [In French]. No 97, October 1997.

20. Garoyan G. One hundred and fourteen years of life, or the long history of Jeanne Calment, France's oldest person [In French]. Marseille: Uiversity of Aix-Marseille, 1990.

21. Bloch K. Blondes in Venetian Paintings, the Nine-Banded Armadillo, and Other Essays in Biochemistry. New Haven, CT: Yale University Press, 1994, p. 13.

22. Cavalie F. Jeanne Calment: "L'oubliee de Dieu." Paris: TF1 Editions, 1995, p. 195.

23. Garrett N, Lazzaro SC, ArielyD, Sharot T. The brain adapts to dishonesty. Nat Neurosci 2016;19:1727-1732.
24. Calment Wiki, fandom.com, Madame Calment. Available at https://calment.fandom.com/wiki/Madame_Calment (accessed June 24, 2019).

25. Hajek A. Dutch book arguments, Chapter 7. In: Anand P, Pattanaik P, Puppe C. (eds): The Oxford Handbook of Rational and Social Choice. Oxford: Oxford University Press, 2009, pp. 173-195.

26. Sprenger J. The Objectivity of Subjective Bayesian Inference. 2016. Available at http://philsci-archive.pitt.edu/11936/1/ ObjectiveBayesianStatistics_v3.pdf (accessed June 24, 2019).

27. Kumar AB, Shamim H, Nagaraju U. Premature graying of hair: Review with updates. Int J Trichol 2018;10:198-203.

28. Keogh EV, Walsh RJ. Rate of greying of human hair. Nature 1965;207:877-878.

29. Guiraud S. Jeanne Calment, the controversy, the mystery, the crazy story! [In French]. Midi Libre, January 23, 2019.

30. Testimony of Didier David, Boyer C. Is longevity hereditary? [in French] La Rev Fr Généalogie 1998;114:24.

31. Sund KL, Rehder CW. Detection and reporting of homozygosity associated with consanguinity in the clinical laboratory. Hum Hered 2014;77:217-224.

32. Horvath S. DNA methylation age of human tissues and cell types. Genome Biol 2013;14:R115.

33. Simonoff G. Jeanne Calment: The Passion for Life. Paris: [in French] Éditions du Rocher, 1995, p. 77.

34. Christen Y. My day with Jeanne, the oldest women in the world [In French]. Figaro, 1995.

35. Huet S. A scientific heritage lost. Neither autopsy, nor sample, but her genome has "probably" been stored [In French]. Libération, August 7, 1997.

36. Johannes F. The Arlesian has gone for always [In French]. Libération, August 7, 1997.

37. Aubenace F. Jeanne Calment, a woman of Arles [In French]. Le Monde, 2019.

Address correspondence to:

Nikolay Zak

Moscow Society of Naturalists

Bolshaya Nikitskaya ulitsa 2

Gerontology Section, Room 9

Moscow 125009

Russia

E-mail: kolyazak@gmail.com

Received: June 25, 2019

Accepted: October 2, 2019 